\title{
Vessels-Sparing Spleen-Preserving Distal Pancreatectomy. Discussion of Management of a Benign Pancreatic Cyst
}

\author{
Mallikarjuna Uppara'* , Adam Esa ${ }^{1}$, Ashraf Rasheed ${ }^{1}$, Mark Robinson ${ }^{2}$, Majid Rashid ${ }^{3}$, Amir Kambal \\ ${ }^{1}$ Gwent Centre for Digestive Diseases, Division of Upper GI Surgery, Royal Gwent Hospital, Newport, UK \\ ${ }^{2}$ Department of Radiology, Royal Gwent Hospital , Newport, UK \\ ${ }^{3}$ Department of Pathology, Royal Gwent Hospital, Newport, Wales, UK \\ ${ }^{4}$ Morriston Hospital, Swansea, UK
}

Received: August 13, 2015; Accepted: November 30, 2015; Published: December 09, 2015

*Corresponding author: Mallikarjuna Uppara, Gwent Centre for Digestive Diseases, Division of Upper GI Surgery, Royal Gwent Hospital, Newport, Wales, UK, Tel: 44-742-744-0407; E-mail: drmallikarjuna@gmail.com

\begin{abstract}
Context: The septic and haematological consequences of splenectomy lead to the development of spleen-preserving techniques during distal pancreatectomy.

Case Report: We report a case of a middle age woman who underwent spleen-preserving distal pancreatectomy for a symptomatic pancreatic cystic lesion. The diagnostic and therapeutic challenges faced when caring for such patients are discussed. The indications for SPDP are visited, different techniques for spleenpreserving pancreatectomy are examined and the rationale behind the chosen technique is explained.
\end{abstract}

Conclusion: Vessels-Sparing, Spleen-preserving distal pancreatectomy is an optimum choice for benign Pancreatic Cystic Lesions.

Keywords: Distal pancreatectomy; SPDP; PCL (Pancreatic Cystic Lesions)

\section{Introduction}

Spleen was thought to play no significant role in adult life and was routinely removed during distal pancreatectomy (1). The transient increase in T-suppressor cells following splenectomy blunts the cellular response to antigens, the decreased IgM production diminishes the anti-body response to antigens not previously encountered, the decrease in properdin and tuftsin levels impairs opsonisation and phagocytosis and increase the risk of overwhelming infection from encapsulated organisms following splenectomy $(1,2)$. This lead to dispelling of the concept of splenectomy as an innocuous procedure and the development of Spleen Preserving Distal Pancreatectomy (SPDP).

We discuss the decision-making process in the case of a middle age women who underwent SPDP examining the evidence supporting these decisions.

\section{Case Report}

A 50 year old female was referred with two months history of severe un-remitting epigastric and back pain. The pain was alleviated but not relieved by opioids. An abdominal UltraSound Study (USS) organised by the referring practitioner revealed a left upper quadrant cystic mass.

Computed Tomography (CT) (Figure 1) confirmed it to be of pancreatic origin measuring $44 \mathrm{~mm}$ in diameter. There were no known risks for, or previous attacks of pancreatitis and all haematological, biochemical, and tumour markers were within normal limits.

The case was reviewed at the specialist Multi-Disciplinary Meeting (MDM) and based on morphology, age and gender, a provisional diagnosis of benign or low grade malignant cyst was entertained. Resection was recommended, and spleenpreserving, pancreatectomy (SPDP) sparing the spleenic artery and vein was carried out (Figures 2).

The procedure lasted just over two hours and a $200 \mathrm{ml}$ blood loss was recorded. The patient had un-eventful recovery and was discharged home on the fifth post postoperative day. Histological examination of pancreatectomy specimen was confirmed to be a pseudocyst (Figure 3).

At 4 weeks review, she had made a full recovery and was back to normal activity. CT images at 1 and 6 months following SPDP were compared with preoperative images to evaluate postoperative changes in vascular patency and to determine whether the analysed vessel was a native or collaterals that developed after surgery following the possible occlusion of the original spleenic artery or vein. The scans confirmed a normal size spleen, patent spleenic vessels and without collateralisation (Figure 3). The patient remained symptoms-free at 9 months and was discharged from surgical follow up.

\section{Discussion}

Pseudocysts are the most common pancreatic cystic lesions. These benign cysts may appear similar to cystic neoplasms and would need to be evaluated by CT, MRI +/- Endo-luminal Ultrasound (EUS). 


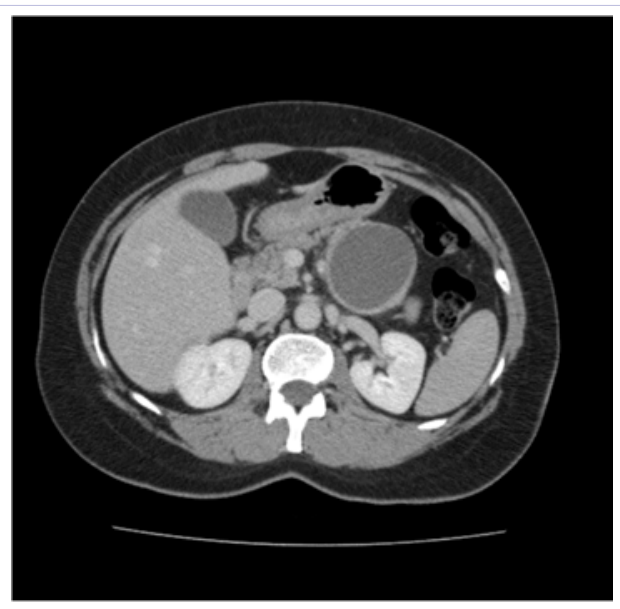

Figure 1: Computed Tomography (CT) of a solitary cystic lesion in the tail of the pancreas. Pancreatic pseudocyst. Axial contrast-enhanced CT images showing a well defined, encapsulated cystic lesion arising from the pancreas.

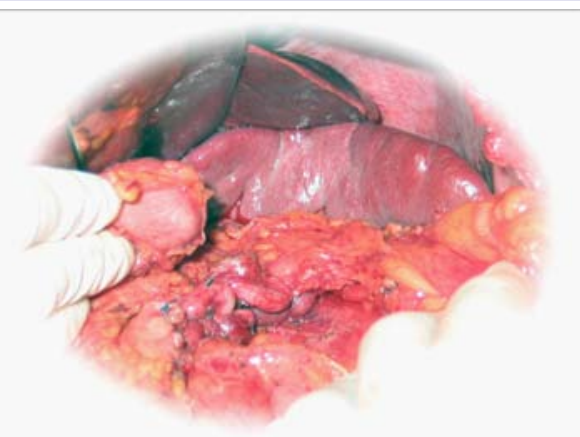

Figure 2: Operative Photograph showing the preserved spleen and the spared splenic vessels.

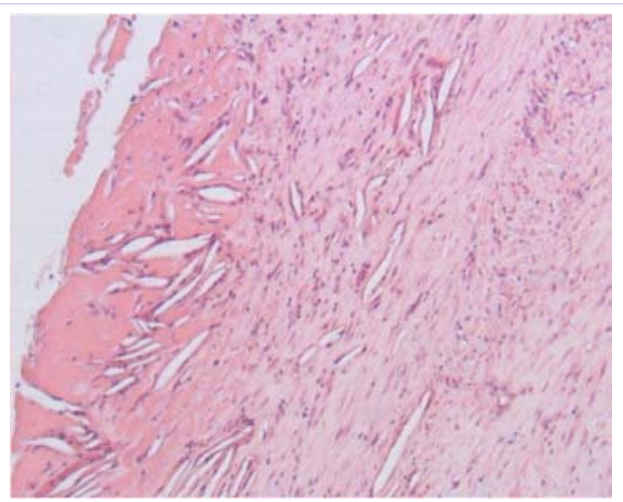

Figure 3: $\mathrm{H} \& \mathrm{E}$ stain illustrating benign cyst wall with organising haemorrhage and no evidence of lining epithelium (Pseudocyst).

\section{The Pre-Operative Evaluation of Pancreatic Cystic lesion}

Cross sectional imaging is limited in its ability to differentiate benign from malignant pancreatic cystic lesions. Papillary projections and peripheral calcification favours malignancy and avid contrast enhancement of the wall or any solid component may point towards cystic endocrine malignancy. MRI is reliable at evaluating the cyst's relationship to the pancreatic-biliary tree and the improved spatial resolution of EUS and its Fine Needle Aspiration (FNA) abilities increased its use especially for small cysts ( $<2 \mathrm{~cm}$ in diameter). EUS is particularly helpful at differentiating the internal architecture of the cyst and delineating its relationship to the spleenic vessels. All image findings should be integrated with demographics and clinical presentation when planning the management strategies for these lesions (3).

\section{Management of Pancreatic Cystic Lesions}

Non-symptomatic, thin-walled, uni-locular cysts, less than 3 $\mathrm{cm}$ in size are commonly benign and a monitoring strategy can be applied safely with 6 monthly imaging for the $1^{\text {st }}$ year followed by annual imaging for a period of 3 years then every 3 years in patients $<70$ years of age. If cyst stability is established and the patient continues to be symptoms-free, no further evaluation may be needed $(3,4)$. We are proposing a flow chart (figure 4 ), which reflects our experience and including our interpretation of current knowledge in management of PCL but this should be applied in the context of a specialist pancreatic MDM's (5).

Surgical resection is, however often indicated for symptomatic and potentially malignant lesions provided the surgical risks are acceptable for the individual patients. Pre-operative EUSguided cyst aspiration for diagnostic and therapeutic purposes was considered in this case. The severe symptoms, lesion's resectability, and absence of extra pancreatic disease in this fit lady, lead to the MDM's recommendation of pancreatic resection without any further testing. This was perceived to be most effective at relieving suffering and reaching a definitive diagnosis.

\section{Pancreatic Resection- The Options}

Distal pancreatic resection can be performed with or without splenectomy and with or without vessels sparing $(6,7)$. The clinical features, preoperative imaging and normal tumour markers in this case suggested the benign nature of the lesion and hence the decision to preserve the spleen (8). This decision starts pre-operatively but completed intra-operatively based on the anatomic relationship of the tail of the pancreas to the splenic hilum, the presence or absence of inflammatory adhesions around the tail of the pancreas or splenic hilum, splenic vein involvement and the extent of resection necessary as determined by the underlying pathology $(4,9)$ and the intra-operative findings.

\section{Techniques for Spleen-Preserving Distal Pancreatec- tomy}

There are two main variations in techniques of SPDP, with or without preservation of splenic artery and vein. In Warshaw's pioneering method, the splenic vessels are ligated at the splenic hilum and spleen becomes dependent on the short gastric vessels (10). This method is technically less demanding and can be accomplished in a shorter operating time and is the preferred technique when the splenic artery and vein are inflamed, fibrosed, thrombosed or involved in the pathological process $(9,10)$. The disadvantages of this method is the association with a higher 


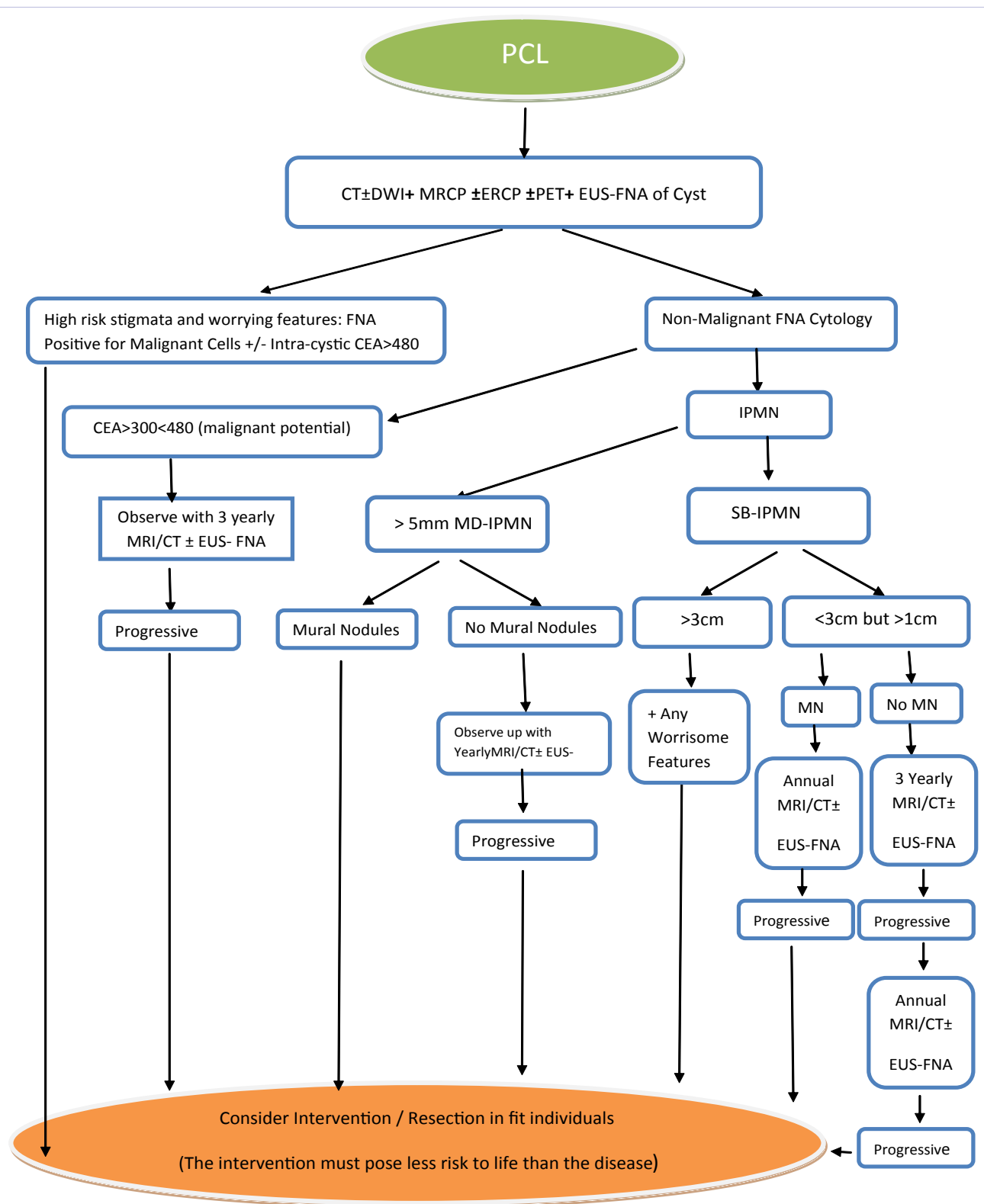

Figure 4: Flow chart.

incidence of spleen vascular insufficiency, such as splenic infarct or necrosis, and of gastric varices and gastrointestinal bleeding possibly due to an increase of venous pressure in the area of the left gastroepiploic and short gastric veins $(8,11)$.

The other technique is the spleen-preserving, vessels-sparing one (our preferred technique) is more demanding and requires meticulous dissection of the pancreas from the splenic vessels. In this technique, a narrow plane (wide enough to accommodate the stapler) is carefully developed between the splenic vein, superior mesenteric vein and the pancreas. The pancreas is then transacted with endopath stapler Echelon 60 (Ethicon Endosurgery; Johnson \& Johnson, Cincinnati, OH, USA) close to but not compromising the first branch of the splenic artery supplying the tip of the pancreas on the remnant side, if the transaction of the pancreas is performed far from this branch, the remnant's tip may become ischemic and lead to pancreatic leak. The cartridge (staple size) is selected according to the thickness and texture of the pancreas (mostly with vascular staples). Additional sutures of $5 / 0$ or $4 / 0$ Prolene is applied as required.

The difficulty in discriminating the distal end of the pancreas from the splenic hilum fat and branching of the splenic vessels as they approach the hilum of the spleen make antegrade pancreatectomy a safer procedure. This is completed by freeing of the pancreatic body from the splenic vein starting at the point of pancreatic transaction and progressing towards the spleen (figure 5). The multiple short vessels connecting the body and tail of the pancreas to the splenic artery and splenic vein are serially isolated, Liga-clipped, oversewn with 5/0 Prolene or divided 
with an ultrasonic shears.

Both techniques of SPDP are achievable laparoscopically (9). However, the degree of encountered retroperitoneal fibrosis makes our selected conventional (open) surgical approach via an upper midline incision with the added benefit of less trauma or luminal compromise to the splenic vein the more appropriate approach for this case. It is however important to remember that while the open technique may give more space to operate and immediate access to the surgical field, the laparoscopic optics magnifies the field making it easier to see and secure the small vessels.

\section{Possible Limitations of SPDP}

Spleen serves as the junction between the mesenteric venous circulation and the oesophagogastric venous plexus. Most of the studies focused on the short-term outcomes of SPDP with little information on the long-term consequences of splenic vessels ligation. When splenic blood is unable to drain through the splenic vein, it flows through the short gastric vessels in a retrograde fashion into the sub-mucosal plexus in the gastric fundus and cardia to drain into the coronary vein. Short term follow up suggests adequacy of the supply by the short gastric and the gastroepiploic arteries; however gastric and perigastric varices were noted on subsequent imaging. In their study during 52 weeks observation, Miura et al concluded that gastric varices located in short gastric veins frequently occurred in patients who underwent SPDP with excision of the splenic vessels leads to a localized venous hypertension called sinistral portal hypertension. The cause of this condition was considered to be the increase of blood flow of gastric veins, but the reason for the increasing gastric venous blood flow despite the absence of splenic arteries is unclear (11).

A single-centre retrospective study evaluated the short and long term patency of preserved splenic vessels after laparoscopic SPDP. A study which included 22 patients confirmed the short term benefit of good perfusion to the spleen. Long term follow up however revealed a risk for left-sided portal hypertension if the splenic vein becomes occluded (12).

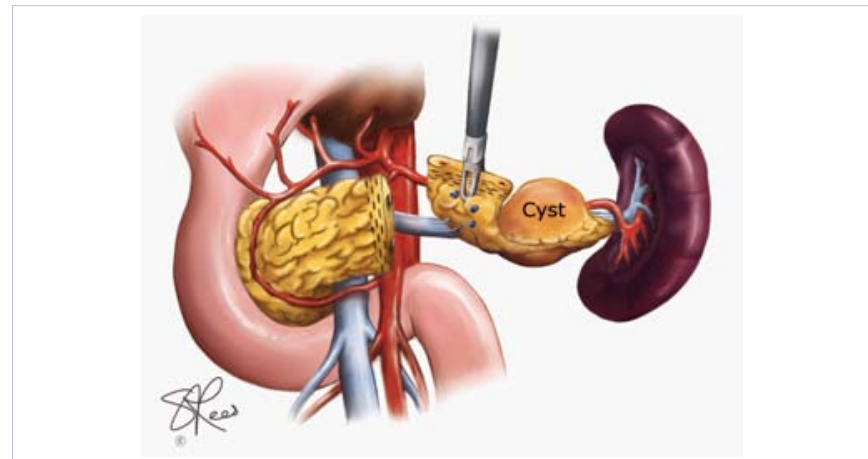

Figure 5: Ante grade pancreatectomy in SPDP (Spleen preserving distal pancreatectomy).
Further follow-up and additional investigations with larger patient populations will be needed before definitive conclusions can be drawn. In any event, we think that the splenic artery and vein should be preserved as far as possible when performing spleen-preserving pancreatectomy.

\section{Conclusion}

Our case report discussed the practical aspect of the procedure without scrutinising the science supporting the practice. The evidence seems to be limited to case series supporting the feasibility and short term safety of SPDP. The procedure seems to have entered the practice without standardisation or being the subject of a trial to evaluate and provide the evidence to this change. We caution against change in the practice based on logic and without any robust scientific support. We would recommend and despite the challenges due to the complexity and limited indications to attempt RCT or as a bare minimum maintain all such cases in a national robust database.

\section{References}

1. Cooper MJ, Williamson RC. Splenectomy: indications, hazards and alternatives. Br J Surg. 1984; 71(3):173-80.

2. Linet MS, Nyrén O, Gridley G, Mellemkjaer L, McLaughlin JK, Olsen $\mathrm{JH}$, et al. Risk of cancer following splenectomy. Int J Cancer. 1996; 66(5):611-6.

3. Byrne MF, Jowell PS. Gastrointestinal imaging: endoscopic ultrasound Gastroenterology. 2002; 122(6):1631-48.

4. Tsuei BJ, Schwartz RW. Current management of pancreatic pseudocysts. Curr Surg. 2003; 60(6):587-90.

5. Mallikarjuna Uppara, Patooru Vijaya kumar, Singaram Palaniappan, Ravi Ramakrishnan, Rajagopal Surendran, Ashraf Rasheed. Clinical, Radiological, Cytological and Biochemical Analysis of Pancreatic Cystic Lesions are Necessary prior to Definitive Therapeutic Planning. JOP. 2015; 16(6):574-581.

6. Jacobson BC, Baron TH, Adler DG, Davila RE, Egan J, Hirota WK, et al. ASGE guideline: The role of endoscopy in the diagnosis and the management of cystic lesions and inflammatory fluid collections of the pancreas. Gastrointest Endosc. 2005; 61(3):363-70.

7. Kleeff J, Diener MK, Z'graggen K, Hinz U, Wagner M, Bachmann J, et al. Distal pancreatectomy: risk factors for surgical failure in 302 consecutive cases. Ann Surg. 2007; 245(4):573-82.

8. Aldridge MC, Williamson RC. Distal pancreatectomy with and without splenectomy. Br J Surg. 1991; 78(8):976-9.

9. Benoist S, Dugué L, Sauvanet A, Valverde A, Mauvais F, Paye F, et al. Is there a role of preservation of the spleen in distal pancreatectomy? J Am Coll Surg. 1999; 188(3):255-60.

10. Fernández-Cruz L, Orduña D, Cesar-Borges G, López-Boado MA. Distal pancreatectomy: en-bloc splenectomy vs spleenpreserving pancreatectomy. HPB (Oxford). 2005; 7(2):93-8. doi: $10.1080 / 13651820510028972$.

11. Shoup M, Brennan MF, McWhite K, Leung DH, Klimstra D, Conlon KC. The value of splenic preservation with distal pancreatectomy. Arch Surg. 2002; 137(2):164-8.

12. Miura F, Takada T, Asano T, Kenmochi T, Ochiai T, Amano H, et al. Hemodynamic changes of splenogastric circulation after spleenpreserving pancreatectomy with excision of splenic artery and vein. 